\title{
SÍNTESIS Y CARACTERIZACIÓN DE POLVOS DE HIDROXIAPATITA CARBONATADA TIPO B CON DIFERENTES CONTENIDOS DE CARBONATO
}

\author{
I. Ochoa ${ }^{1 *}$, E. López ${ }^{2}$, H. Copete ${ }^{3}$
}

1: Ingeniera de Materiales, Universidad de Antioquia. Medellín, Colombia

2: Ph. D. en Ciencia y Tecnología de Materiales, Universidad de Oviedo. Oviedo, España

3: M.Sc. Ingeniero de Materiales, Universidad de Antioquia. Medellín, Colombia

*maria.ochoag@udea.edu.co

\begin{tabular}{|c|c|}
\hline PALABRAS CLAVE & RESUMEN \\
\hline $\begin{array}{l}\text { Hidroxiapatita Carbonatada } \\
\text { tipo B } \\
\text { Hidroxiapatita } \\
\text { Precipitación vía húmeda } \\
\text { Método inverso }\end{array}$ & $\begin{array}{l}\text { La Hidroxiapatita (HA) es un material ampliamente utilizado en aplicaciones } \\
\text { biológicas, aunque su aplicación en muchas ocasiones se limita debido a su } \\
\text { estabilidad en ambientes fisiológicos. Es por esto que surge como alternativa, la } \\
\text { Hidroxiapatita Carbonatada tipo B (HAC), la cual posee mayor similitud al } \\
\text { hueso biológico debido a la presencia del ion carbonato en su estructura. Para su } \\
\text { obtención se han utilizado distintos métodos de síntesis, sin embargo, la } \\
\text { complejidad y poca reproducibilidad de muchos ha llevado a que sea un tema } \\
\text { cada vez más investigado. En este trabajo se muestra la síntesis y caracterización } \\
\text { de la HA y la HAC tipo B por el método de precipitación en vía húmeda de tipo } \\
\text { inverso, con } 3 \text { contenidos de carbonato diferentes, denotados como C2, C3 y C4 } \\
\text { con relaciones carbono/fósforo (C/P) de } 0,25 \text {, } 0,375 \text { y } 0,5 \text { respectivamente, } \\
\text { siendo C4 la HAC con mayor contenido de carbonato. Los polvos sintetizados } \\
\text { se caracterizaron mediante difracción de rayos X (DRX), fluorescencia de rayos } \\
\text { X de longitud de onda dispersiva (FRX-OD), combustión de carbono mediante } \\
\text { analizador Leco, espectroscopia infrarroja con transformada de Fourier (FTIR) } \\
\text { y microscopia electrónica de transmisión (TEM). }\end{array}$ \\
\hline
\end{tabular}

\section{SYNTHESIS AND CHARACTERIZATION OF TYPE B CARBONATE HYDROXYAPATITE POWDERS WITH DIFFERENT CARBONATE CONTENTS}

\begin{tabular}{|c|c|}
\hline KEYWORDS & ABSTRACT \\
\hline $\begin{array}{l}\text { Carbonated hydroxyapatite } \\
\text { type B } \\
\text { Hydroxyapatite } \\
\text { Wet precipitation } \\
\text { Reverse method }\end{array}$ & $\begin{array}{l}\text { Hydroxyapatite (HA) is a material widely used in biological applications, } \\
\text { although its application is often limited due to its stability in physiological } \\
\text { environments. That is why it arises as an alternative, the Carbonate } \\
\text { Hydroxyapatite type B (CHA), which has greater similarity to biological bone } \\
\text { due to the presence of carbonate ion in its structure. In order to obtain it, different } \\
\text { synthesis methods have been used, however, the complexity and low } \\
\text { reproducibility of many of them has made it an increasingly researched topic. In } \\
\text { this work it is shown the synthesis and characterization of HA and CHA type B } \\
\text { by the inverse type wet precipitation method, with } 3 \text { different carbonate contents, } \\
\text { denoted as C2, C3 and C4 with carbon/phosphorus relations (C/P) of } 0.25,0.375 \\
\text { and } 0.5 \text { respectively, being C4 the CHA with higher carbonate content. The } \\
\text { synthesized powders were characterized by X-ray diffraction (XRD), X-ray } \\
\text { fluorescence (WD-XRF), combustion of carbon by Leco analyzer, Fourier } \\
\text { transform infrared spectroscopy (FTIR) and transmission electron microscopy } \\
\text { (TEM). }\end{array}$ \\
\hline
\end{tabular}




\section{INTRODUCCIÓN}

El desarrollo de nuevos materiales con características específicas para ciertas aplicaciones ha sido un reto en el avance científico de nuevos materiales para diversas áreas, entre los cuales se encuentra la medicina [1]. Desde hace tiempo muchas investigaciones se centran en materiales que puedan reemplazar las partes del cuerpo humano, para lo cual se buscan características físicas, químicas, biológicas y mecánicas muy similares al lugar que se desea implantar [1]; dentro de ellos se encuentran los injertos óseos, que componen el segundo trasplante de tejido más frecuente en todo el mundo después de la transfusión de sangre, además es uno de los procedimientos quirúrgicos más utilizados para el aumento de la regeneración ósea en los procedimientos ortopédicos, se indica que anualmente se realizan más de dos millones de procedimientos de injertos óseos en todo el mundo [2].

Debido al crecimiento poblacional también aumentaron la cantidad de defectos óseos tanto congénitos como ocasionados por traumatismos, secuelas oncológicas e infecciosas, lo que llevó a que muchos científicos enfocaran sus investigaciones en implantes a base de materiales que ayudaran a restablecer la integridad anatómica y funcional de la estructura alterada, para esto las investigaciones se centraron cada vez más en el desarrollo de materiales cerámicos con características similares a las del sistema óseo [3].

Los fosfatos de calcio, principalmente la Hidroxiapatita Estequiométrica $\mathrm{Ca}_{10}\left(\mathrm{PO}_{4}\right)_{6}(\mathrm{OH})_{2} \quad(\mathrm{HA}) \quad$ con relación $\mathrm{Ca} / \mathrm{P}=1,67$, es uno de los materiales más utilizados en la ingeniería biomédica debido a su similitud con la fase mineral del hueso. Dado que es muy estable químicamente y tiene una estructura cristalina muy rígida, posee una cinética de reabsorción muy baja en medio fisiológico y presenta bajas propiedades mecánicas lo que limita su aplicación [3], por lo tanto, el desarrollo de biomateriales a base de fosfatos de calcio se enfoca en encontrar implantes que interactúen rápidamente con el ambiente biológico circundante y que posea las características mecánicas necesarias para soportar la carga a la cual está sometida la parte del sistema óseo que se desea reemplazar.

Durante muchos años, las fases minerales de los tejidos calcificados se habían idealizado como Hidroxiapatita pura, sin embargo, Legeros demostró por primera vez que la apatita biológica no es HA pura, sino que es una apatita carbonatada similar a la apatita sintética con sustituciones del grupo carbonato $\left(\mathrm{CO}_{3}{ }^{2-}\right)$ por el ion fosfato $\left(\mathrm{PO}_{4}{ }^{3-}\right)$ [4] como se muestra en la Figura 1.

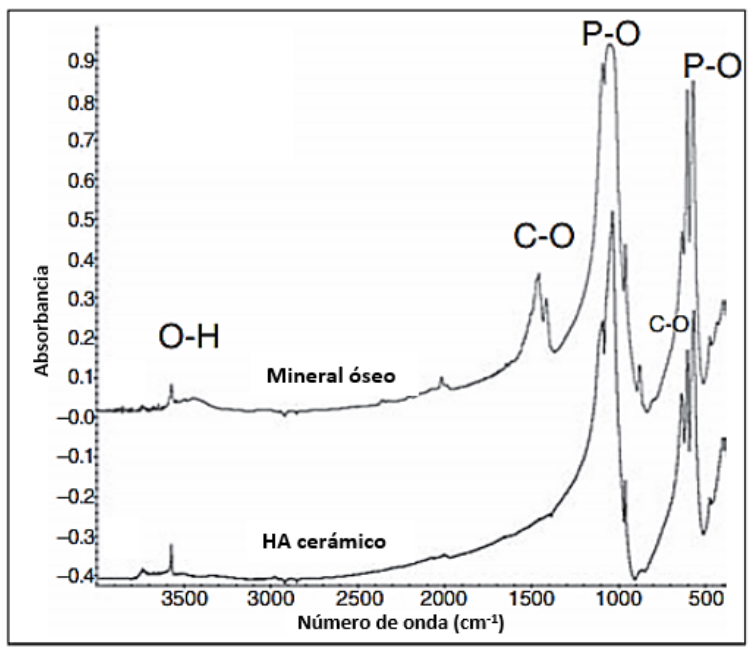

Figura 1. Espectros FTIR de la HA cerámico y el mineral óseo. Modificada de [4].

En la Hidroxiapatita los iones hidroxilo $(\mathrm{OH})$ y fosfatos $\left(\mathrm{PO}_{4}{ }^{3-}\right)$ pueden ser sustituidos por iones carbonato $\left(\mathrm{CO}_{3}^{2-}\right)$, obteniendo dos tipos de Hidroxiapatita Carbonatada (HAC) que se clasifica como tipo A cuando el $\mathrm{CO}_{3}{ }^{2-}$ ocupa el lugar de los iones $\mathrm{OH}^{-}$y tipo $\mathrm{B}$ cuando el $\mathrm{CO}_{3}{ }^{2-}$ reemplaza los iones $\mathrm{PO}_{4}{ }^{3-}$ como se muestra en la Figura 2. También se puede 
presentar los dos tipos de sustitución al mismo tiempo, denominada tipo $\mathrm{AB}$ [5]. Se ha encontrado que la HAC tipo B es la que conforma principalmente el hueso humano y debido a la aparición de grupos activos en su estructura posee importantes reportes en propiedades de biodegradabilidad, biocompatibilidad, osteoinducción, osteoconducción y propiedades que hacen a este biomaterial con un gran potencial para su uso en la ingeniería biomédica [3], lo que la convierte en un material con alto potencial para aplicaciones como injerto óseo.

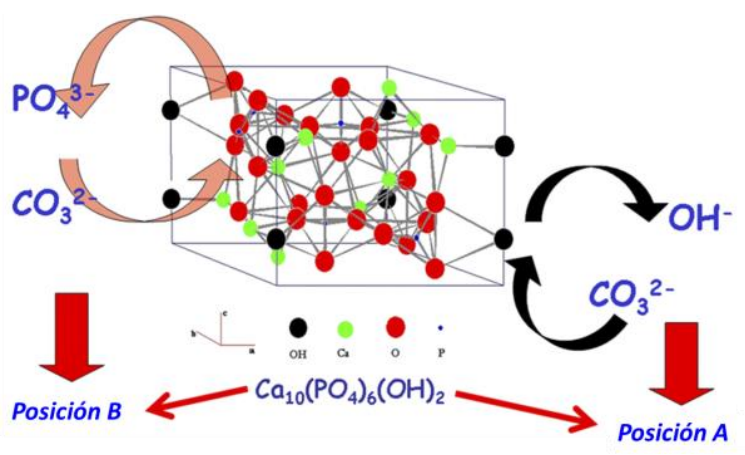

Figura 2. Sustitución Iónica de la Hidroxiapatita [6].

La Hidroxiapatita Carbonatada tipo B cristaliza en el sistema hexagonal y es isomorfa a la Hidroxiapatita sin sustituir, en este caso las sustituciones de los iones fosfato por los iones carbonato generan diversas perturbaciones en la red de la Hidroxiapatita que provoca la disminución de la cristalinidad, menor estabilidad térmica y un aumento de la solubilidad del biocerámico tanto en las pruebas in vitro como in vivo; además, se ha demostrado una mayor afinidad de las células osteoblásticas (células encargadas de la regeneración ósea), en comparación con la HA debido a características intrínsecas del material como su baja cristalinidad y menor estabilidad química lo que genera una mayor afinidad biológica [7]
En la Hidroxiapatita Carbonatada tipo B los iones $\mathrm{CO}_{3}{ }^{2-}$ reemplazan los grupos $\mathrm{PO}_{4}{ }^{3-}$ como se muestra a continuación en la Fórmula Estequiométrica [1].

$$
\left.\mathrm{Ca}_{10-\mathrm{x}}\left(\mathrm{PO}_{4}\right)_{6-\mathrm{x}}\left(\mathrm{CO}_{3}\right)_{\mathrm{x}}\right)(\mathrm{OH})_{2-\mathrm{x} ;} 0 \leq \mathrm{x} \leq 2
$$

Donde " $x$ " es la cantidad de iones carbonato que sustituyen los iones $\mathrm{PO}_{4}{ }^{3-}$, donde se puede observar que estequiométricamente en este tipo de Hidroxiapatita Carbonatada también se da en menor grado la sustitución del grupo $\mathrm{OH}^{-}$por el ion carbonato, algo que es difícilmente controlable durante el proceso de síntesis [5].

Sintetizar el polvo es sin duda el paso inicial en el proceso de fabricación de un implante, la síntesis debe ser lo más sencilla y reproducible posible. La preparación de la Hidroxiapatita Carbonatada tipo B sigue siendo cuestionable ya que es poco reproducible [8] debido a que se deben controlar muchos parámetros como la temperatura, la atmósfera, las cantidades y pureza de los precursores, el $\mathrm{pH}$, entre otros, para lograr obtener Hidroxiapatitas Carbonatadas tipo B con las características químicas, físicas, mecánicas y biológicas aptas para ser aplicados como implantes óseos. Por ejemplo la relación $\mathrm{Ca} / \mathrm{P}$, pureza y nivel de carbonatación (por mencionar algunos) deben ser lo más similares posibles a la Hidroxiapatita biológica para que el material pueda ser implantado sin generar efectos adversos en la persona. En gran parte la importancia de sintetizar HAC con tasas de carbonatación definidas, radica en que el contenido de carbonato en la parte mineral del hueso es de aproximadamente 4-8 \% en peso, este contenido puede variar dependiendo la edad de la persona, por lo que el método de síntesis es fundamental para obtener una HAC con las características óptimas para su aplicación en la ingeniería biomédica [5]. 
Entre los métodos utilizados para la síntesis de la HAC se encuentran la precipitación en húmedo, el proceso hidrotermal, la síntesis por sol-gel, el método mecanoquímico, entre otros. El método de síntesis utilizado define en gran parte la eficacia del material para poseer las propiedades mecánicas, físicas, químicas y biológicas necesarias para su utilización en aplicaciones médicas [9]. Entre los métodos más utilizados para la síntesis de la HAC tipo B se encuentra el proceso hidrotermal, el cual se realiza en vía húmeda, a alta temperatura y presión; no obstante, la tasa de carbonatación por este método es bajo, de aproximadamente el $1 \%$ en masa y el tiempo de síntesis suele ser mayor a las 24 horas [10], por tanto su aplicación se reduce, por lo que el método de precipitación húmeda es de los más utilizados actualmente

Legeros y otros [11], desarrollaron un método sencillo y fácilmente reproducible para la síntesis de la HAC tipo B que consistía en adicionar lentamente una solución de acetato de calcio a una solución con fosfato diamónico y carbonato monosódico (Figura 3a); este método de síntesis fue denominado método directo y se realiza bajo una temperatura de $95{ }^{\circ} \mathrm{C}$ en 1,5 horas. Luego el material precipitado es filtrado y lavado con agua destilada y finalmente secado hasta eliminar la humedad y los residuos de la síntesis; el inconveniente de este método es que el uso del carbonato monosódico proporciona una alta concentración en la solución de iones sodio, que posteriormente pueden precipitar como ion alcalino en el material, cambiando su composición química $[5,11,12]$.

Vignoles y otros [13], con el fin de conseguir HAC tipo B sin la presencia de iones alcalinos propusieron reemplazar el carbonato de sodio por carbonato de amonio y realizar el procedimiento de tipo inverso (Figura 3b). Este método consiste en agregar mediante goteo a la solución que contiene los iones calcio, la solución con los iones fosfato y carbonato; evitando que los iones amonio provenientes del carbonato de amonio se formen una vez entren en contacto con la solución de calcio $[5,13]$. Este método es el más utilizado en la fabricación de Hidroxiapatitas Carbonatadas tipo B debido a que se evita la presencia de iones alcalinos en la composición química.

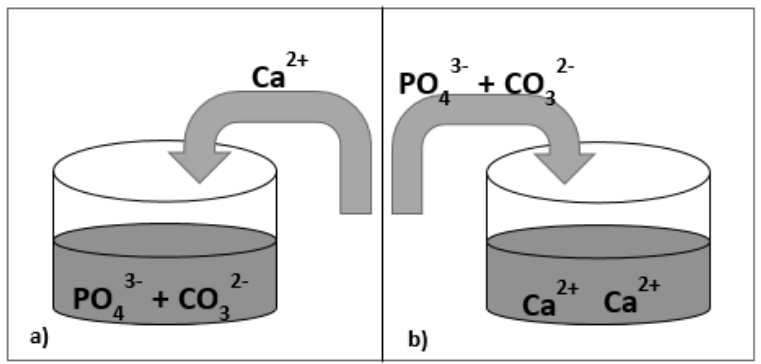

Figura 3. Sintesis Hidroxiapatita Carbonatada tipo B a) método directo b) método inverso.

Por otro lado, Lafon (2004) [8] realizó un trabajo donde sintetizaba Hidroxiapatitas Carbonatadas tipo B utilizando el método inverso, demostrando que la tasa de carbonatación (relación molar $\mathrm{CO}_{3}{ }^{2-} / \mathrm{PO}_{4}{ }^{3-}$ ), el $\mathrm{pH}$, la temperatura, la atmósfera, la concentración y pureza de los reactivos (relación $\mathrm{Ca} / \mathrm{P}$ ) eran los parámetros que definían la naturaleza y cristalinidad de la de HAC-B. Los reactivos utilizados por el autor para los precursores de los iones calcio, carbonato y fosfato fueron el nitrato de calcio $\mathrm{Ca}\left(\mathrm{NO}_{3}\right)_{2}$. El bicarbonato de amonio $\left(\mathrm{NH}_{4}\right) \mathrm{HCO}_{3}$ y el fosfato diamónico $\left(\mathrm{NH}_{4}\right)_{2} \mathrm{HPO}_{4}$ respectivamente, y así poder garantizar durante toda la síntesis la relación molar $\mathrm{Ca} / \mathrm{P}$ y demostrando con esta investigación la eficacia y versatilidad del método inverso, por lo que es el método de síntesis elegido en este trabajo. [5, 8]

\section{METODOLOGÍA EXPERIMENTAL}

La síntesis de la Hidroxiapatita Carbonatada se realizó siguiendo los parámetros 
estandarizados en un protocolo fabricado por el grupo GIMACYR (Grupo de Investigación en Materiales y Recubrimientos Cerámicos) de la Universidad de Antioquia, MedellínColombia, el cual está basado en lo expuesto por Lafon en 2004 [8].

La síntesis de la HAC tipo B se inicia con la disolución del nitrato de calcio $\left(\mathrm{Ca}\left(\mathrm{NO}_{3}\right)_{2}\right) \cdot 4 \mathrm{H}_{2} \mathrm{O}-\left(\right.$ Merck, p.a. EMSURE ${ }^{\circledR}$ ACS)) en agua destilada en un vaso reactor con agitación magnética constante; una vez disuelto se cierra el reactor (Figura 4a) y se ingresa a un baño de silicona para mantener la temperatura constante y se comienza el calentamiento hasta $95{ }^{\circ} \mathrm{C}$. De manera paralela se disuelven el fosfato diamónico $\left(\mathrm{NH}_{4}\right)_{2} \mathrm{HPO}_{4}$ en agua destilada y se adiciona el bicarbonato de amonio $\left(\mathrm{NH}_{4}\right) \mathrm{HCO}_{3}$. $\mathrm{La}$ cantidad dependerá de la tasa de carbonatación deseada, de acuerdo a la relación carbono/fósforo como se muestra en la Tabla 1. En este trabajo se evaluaron 3 cantidades diferentes de bicarbonato de amonio denotadas como $\mathrm{C} 2, \mathrm{C} 3$ y $\mathrm{C} 4$ siendo C4 la HAC tipo B con mayor contenido de carbonato, además se comparó con Hidroxiapatita como blanco.

Tabla 1. Relación molar carbono/fósforo (C/P) para la sintesis de Hidroxiapatita Carbonatada.

\begin{tabular}{|c|c|}
\hline Notación & $\begin{array}{c}\text { Relación } \\
\text { molar }(\mathbf{C} / \mathbf{P})\end{array}$ \\
\hline $\mathrm{C} 2$ & 0,25 \\
\hline $\mathrm{C} 3$ & 0,375 \\
\hline $\mathrm{C} 4$ & 0,5 \\
\hline
\end{tabular}

Posteriormente se inicia el flujo de argón para asegurar una atmósfera inerte durante la reacción de síntesis para controlar el contenido de carbonato en la Hidroxiapatita, además se adiciona una solución de amoniaco para aumentar el pH de la solución a un valor mayor a 9 para prevenir la aparición de fases secundarias. A continuación, se inicia el goteo de la solución que contiene los iones fosfato y carbonato hasta que se agote por completo (Figura 4b). A continuación, se sostienen las condiciones de temperatura, agitación y atmósfera inerte durante $30 \mathrm{~min}$, se deja enfriar para que se asiente el precipitado que se muestra en la Figura 4c, se filtra y lava con agua destilada. Finalmente el precipitado obtenido se seca durante $12 \mathrm{~h} \mathrm{a}$ una temperatura de $80{ }^{\circ} \mathrm{C}$ en una estufa de secado (Figura 4d).

La caracterización de los polvos para conocer la composición química y las fases presentes, se llevó a cabo por medio de difracción de rayos $\mathrm{X}$ (DRX), fluorescencia de rayos $\mathrm{X}$ por longitud de onda dispersiva (FRX-OD), análisis de carbono por combustión utilizando el analizador Leco y espectroscopia infrarroja con transformada de Fourier (FTIR).

La difracción de rayos X (DRX) se llevó a cabo utilizando un Difractómetro de Rayos X Malvern-PANalytical Modelo Empyean 2012, con detector Pixel 3D y fuente de $\mathrm{Cu}$ $(\lambda=1,541874)$ a $45 \mathrm{kV}$ y $40 \mathrm{~mA}$, con paso de $0,02^{\circ}$ y un tiempo por paso de $46 \mathrm{~s}$. Por su parte la fluorescencia de rayos $\mathrm{X}$ de longitud de onda dispersiva (FRX-OD) se evaluó en un espectrómetro Thermo Scientific empleando fuente de Rodio $(\mathrm{Rh})$ y el análisis de carbono elemental se llevó a cabo en un analizador Leco 527. La espectroscopia de infrarroja con transformada de Fourier (FTIR) en un equipo PelkinElmer y utilizando sistema de reflectancia total atenuada ATR, haciendo un barrido en número de onda entre 4000 y 450 $\mathrm{cm}^{-}{ }^{1}$. Morfológicamente los polvos se caracterizaron mediante microscopia electrónica de transmisión (TEM) en un Microscopio Electrónico de Transmisión Tecnai F20 Super Twin TMP de FEI, usando campo claro y un voltaje de $200 \mathrm{KeV}$. 


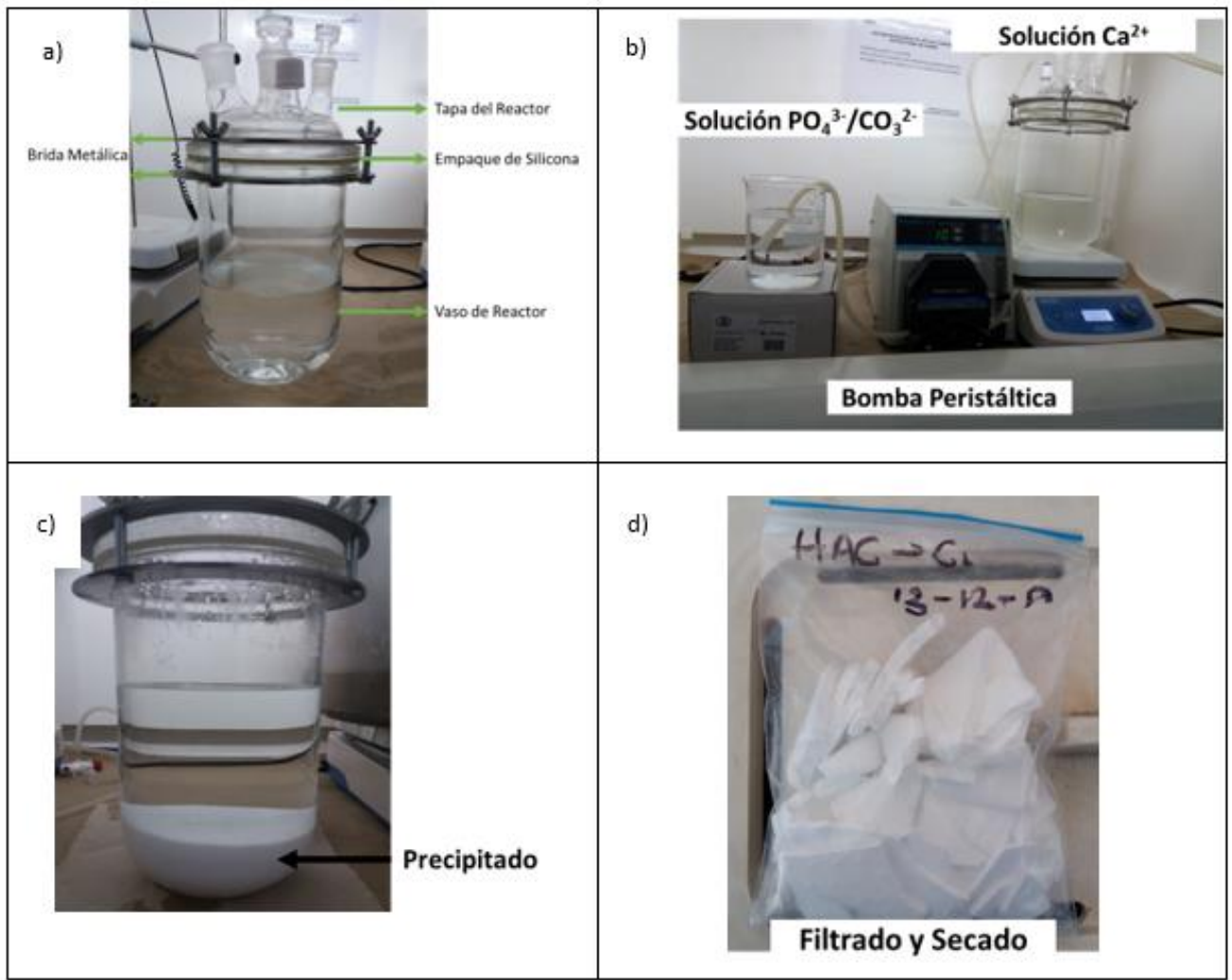

Figura 4. Proceso de síntesis de la Hidroxiapatita. a) disolución de nitrato de calcio y armado del reactor, $\boldsymbol{b})$ adición por goteo de fosfato y carbonato de amonio, $\boldsymbol{c}$ ) precipitado luego de maduración, $\boldsymbol{d}$ ) material después de filtrado y secado.

\section{RESULTADOS Y ANÁLISIS}

Los espectros FTIR de la muestra en polvo de Hidroxiapatita, la cual no posee ningún contenido de carbonato y la Hidroxiapatita Carbonatada con mayor contenido de carbonato $\mathrm{C} 4$ y con relación $\mathrm{C} / \mathrm{P}$ de 0,5 se muestra en la Figura 5, donde se puede observar que las bandas correspondientes al $\mathrm{CO}_{3}{ }^{-2}$ son significativamente intensas para Hidroxiapatita Carbonatada tipo C4 mientras que en la Hidroxiapatita estas bandas no están presentes. La región del espectro para las muestras con carbonato que representan la característica de la Hidroxiapatita Carbonatada tipo B son las vibraciones de $\mathrm{CO}_{3}{ }^{2-}$ detectadas a $871-873 \mathrm{~cm}^{-1}\left(v_{2}\right) \mathrm{y}$ $1416-1486 \mathrm{~cm}^{-1}\left(v_{3}\right)$ tal y como se muestra en la Figura 5 [14], confirmando que la síntesis se realizó de manera eficaz ya que se logró obtener HAC tipo B y no hay presencia de sustitución tipo A, la cual aparece en los rangos $878-888$ y $1500-1550 \mathrm{~cm}^{-1}$. Se puede observar que la HA tiene un pico agudo en aproximadamente $\quad 3570 \mathrm{~cm}^{-1}$ que corresponde a la vibración de los iones $\mathrm{OH}^{-}$, mientras que para la muestra $\mathrm{C} 4$ esta banda tiene menor intensidad, lo que sugiere que hay una menor cantidad de grupos $\mathrm{OH}^{-}$para la Hidroxiapatita Carbonatada tipo C4, esto se debe a que el ion Carbonato pudo haber sustituido parte de los iones $\mathrm{OH}^{-}$, algo que es inevitable durante la síntesis de la Hidroxiapatita Carbonatada tipo B y que estequiometricamente se puede observar en la Fórmula Estequiométrica [1].

La banda atribuible al agua absorbida se presenta en aproximadamente $1640 \mathrm{~cm}^{-1}$ lo cual es una característica típica para la HA, 
pero no para $\mathrm{C} 4$ donde se puede observar menor intensidad de esta banda. Las bandas características de los grupos $\mathrm{PO}_{4}{ }^{3-}$ de la estructura de apatita se observaron aproximadamente en 566-568 y 603$604 \mathrm{~cm}^{-1}\left(v_{4}\right)$ y $1039-1046 \mathrm{~cm}^{-1}\left(v_{3}\right)$ para las dos muestras [14].

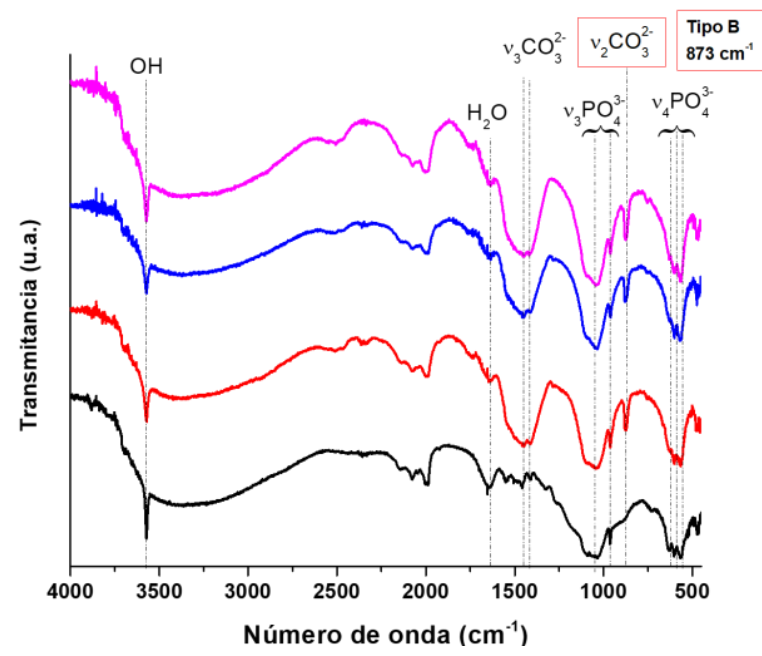

Figura 5. Espectroscopia infrarroja con transformada de Fourier (FTIR) de la Hidroxiapatita (HA) y la Hidroxiapatita Carbonatada tipo $C 4$.

La Figura 6 muestra los resultados de la difracción de rayos $\mathrm{X}$ de la Hidroxiapatita (HA) y la Hidroxiapatita Carbonatada con diferentes contenidos de Carbonato. Los picos presentes en todas las muestras son representativos para la Hidroxiapatita, además, no se presentaron fases secundarias y se detectaron 10 picos de los cuales 6 son de baja intensidad, el primero a $26^{\circ}$ que corresponden a la orientación (002) y otro a $28^{\circ}$ de (210), picos más amplios y superpuestos de (211), (112), (300) y (200) a $32^{\circ}-34^{\circ}$, uno alrededor de $40^{\circ}$ (310) y planos característicos (222), (213) y (004) ubicados a $47^{\circ}, 49^{\circ}$ y $53^{\circ}$, respectivamente [14].

Se puede observar que el aumento del contenido de carbonato provoca un ensanchamiento de los picos lo que representa una disminución de la cristalinidad de la Hidroxiapatita al aumentar el contenido de carbonato, desde la HAC C2 con relación molar C/P 0,25 hasta la HAC C4 la cual posee el mayor contenido de carbonato de las muestras evaluadas y relación molar $\mathrm{C} / \mathrm{P}$ de 0,5 . Esto se debe a que en la HA al ser una celda hexagonal compacta la sustitución del ion carbonato en la estructura modifica el parámetro de red, lo que genera una baja cristalinidad ya que, al sustituir el ion $\mathrm{PO}_{4}{ }^{3-}$ con el ion $\mathrm{CO}_{3}{ }^{-2}$ se induce a la contracción del eje a y expansión en el eje c, por lo tanto se genera un incremento de la relación de los parámetros de red c/a [15], lo que ha demostrado mejorar la reabsorción de la Hidroxiapatita Carbonatada [7]. Además, se puede verificar que no se formó ninguna fase secundaría durante la síntesis de la HA y la HAC con distintos porcentajes de carbonato.

Las tablas 2 y 3 muestran las relaciones molares de $\mathrm{Ca} / \mathrm{P}$ y el porcentaje de carbonato en cada una de las muestras, los cuales fueron calculados a partir de los resultados del análisis químico realizado mediante fluorescencia de rayos X (FRX-OD) y por combustión carbono mediante analizador Leco. En la Tabla 2 se puede observar que la relación $\mathrm{Ca} / \mathrm{P}$ para la $\mathrm{HA}$ es de 1,49 y que esta relación crece al aumentar el contenido de carbonato en la Hidroxiapatita, esto se debe a que a medida que el ion $\mathrm{CO}_{3}{ }^{2-}$ sustituye el $\mathrm{PO}_{4}{ }^{3-}$, la cantidad de fósforo en la estructura disminuye y por ende la relación $\mathrm{Ca} / \mathrm{P}$ aumenta. Varias investigaciones afirman que la utilización de fosfatos de calcio con relaciones $\mathrm{Ca} / \mathrm{P}$ entre 1,4 y 2 son aptos para su aplicación como implante óseo, lo que confirma que la HA y los distintos tipos de HAC sintetizadas en el trabajo pueden ser potencialmente aptos como materia prima para implantes $[16,17,4]$ 


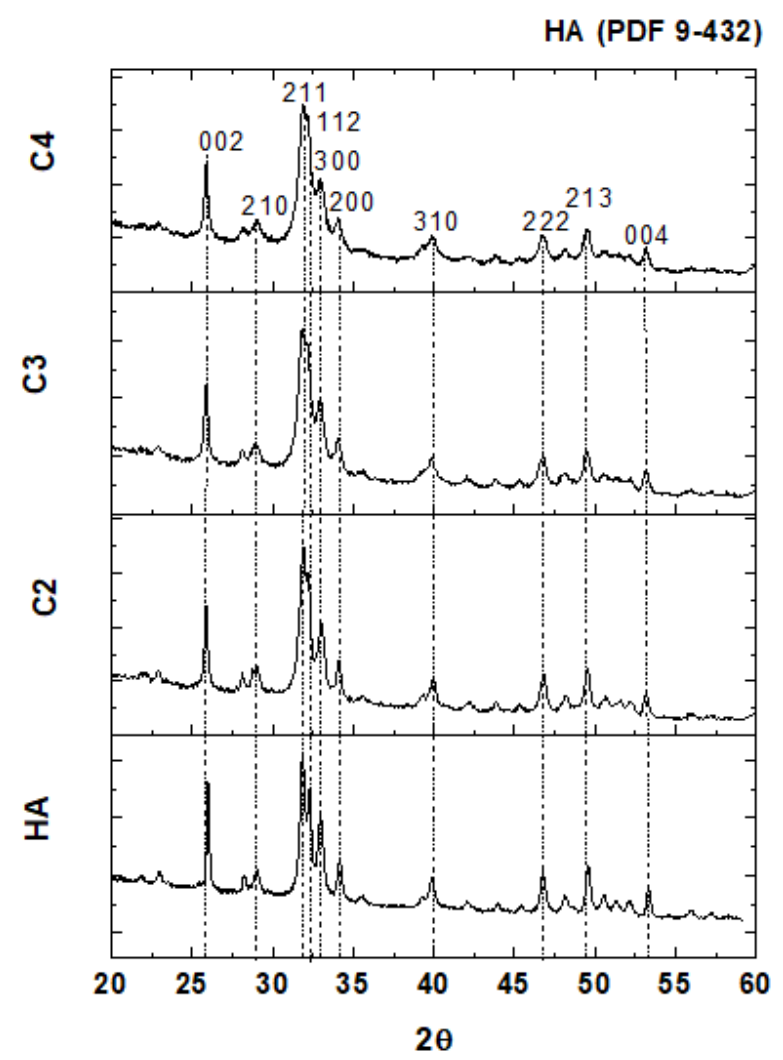

Figura 6. Difracción de rayos $X$ de la Hidroxiapatita (HA) y los diferentes tipos Hidroxiapatita Carbonatada.

Tabla 2. Relación molar Ca/P de la Hidroxiapatita y los diferentes tipos de Hidroxiapatita Carbonatada.

\begin{tabular}{|c|c|}
\hline \multicolumn{2}{|c|}{ Relación molar Ca/P } \\
\hline HA & 1,49 \\
\hline C2 & 1,71 \\
\hline C3 & 1,72 \\
\hline C4 & 1,73 \\
\hline
\end{tabular}

La Tabla 3 muestra el porcentaje en peso de carbonato para los diferentes materiales, donde se puede verificar que la cantidad de carbonato para los distintos tipos de HAC se encuentran en el rango de 4 a $8 \%$ en peso, este es el rango de contenido de carbonato en la parte mineral del hueso [5]. La cantidad de carbonato y la relación $\mathrm{Ca} / \mathrm{P}$ puede variar dependiendo la edad de la persona $\mathrm{y}$ características propias del organismo. Se puede decir entonces, que los polvos sintetizados pueden ser aptos para su utilización en la biomedicina.

Tabla 3. Contenido de carbonato calculado a partir del análisis de carbono.

\begin{tabular}{|c|c|}
\hline Muestra & $\begin{array}{c}\text { Carbonato (\% en } \\
\text { peso) }\end{array}$ \\
\hline HA & 0 \\
\hline C2 & 4,1 \\
\hline C3 & 4,25 \\
\hline C4 & 5,9 \\
\hline
\end{tabular}

Las imágenes de microscopia electrónica de transmisión de campo brillante de las partículas de HA y HAC C4 se muestran en la Figura 7; se puede observar que la HA posee una morfología similar a la de un bastón o aguja y muestran menos tendencia a aglomerarse en comparación con la HAC C4, la cual presenta una morfología más equiaxial y una mayor aglomeración. De acuerdo con lo reportado en la literatura esto se debe a la distorsión en la red que genera las sustituciones del ion carbonato por el ion fosfato generando una leve disminución de la cristalinidad del material y que se pudo observar en los resultados de DRX [18, 19]. 


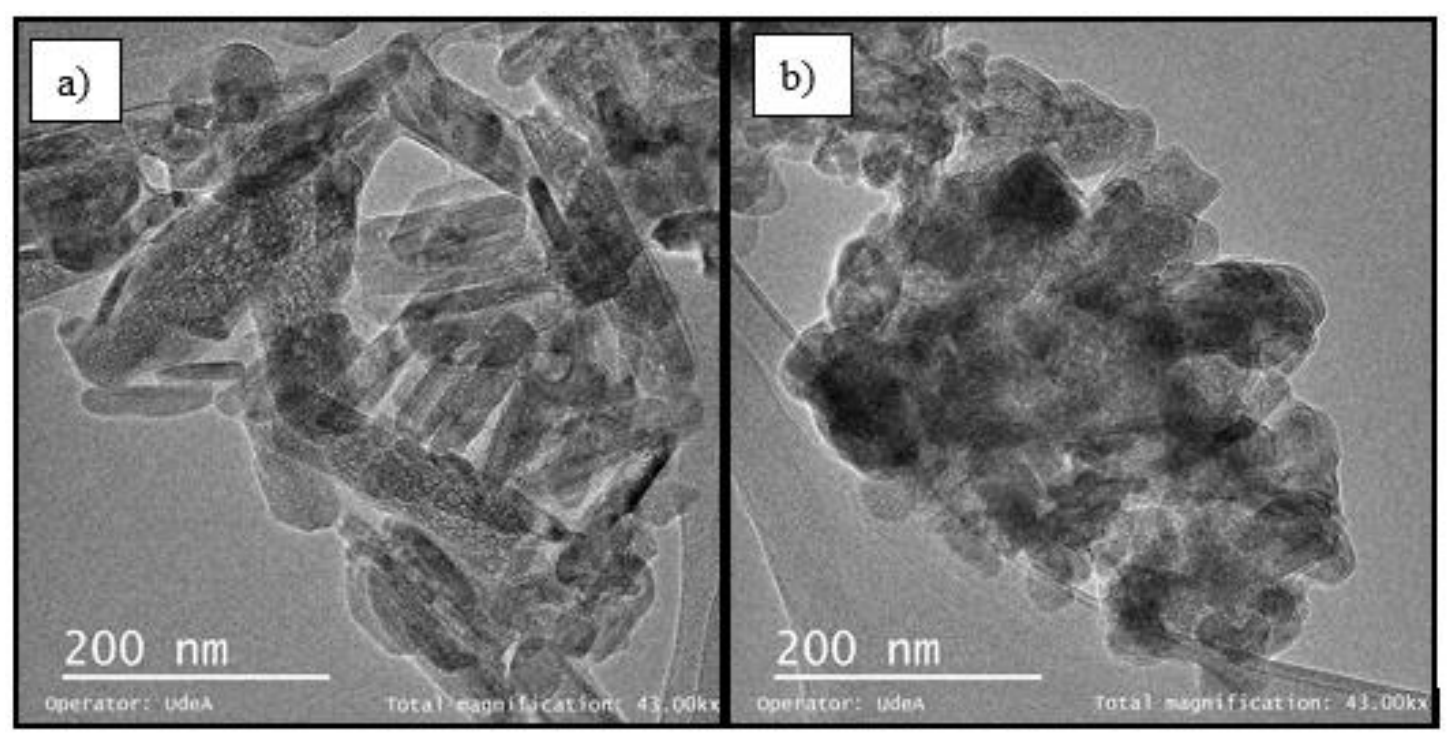

Figura 7. Imágenes TEM a) Hidroxiapatita (HA) b) Hidroxiapatita Carbonatada tipo C4.

\section{CONCLUSIONES}

Durante el trabajo se logró sintetizar Hidroxiapatita e Hidroxiapatita Carbonatada tipo B con diferentes contenidos de carbonato por el método de precipitación por vía húmeda de tipo inverso, sin la presencia de fases secundarias. Además, se encontró que la única fase presente es Hidroxiapatita en todos los materiales sintetizados y un ensanchamiento de los picos al incluir carbonato, el cual produce distorsión en la red cristalina al sustituir el ion fosfato, lo que a su vez generó un cambio en la morfología de los polvos obtenidos, siendo la morfología de la HA bastones definidos y poco aglomerados a una morfología equiaxial y polvos aglomerados para a HAC.

Se demostró que la cantidad de carbonato para los distintos tipos de Hidroxiapatita Carbonatada se encuentran en el rango que posee la parte mineral del hueso (4 a $8 \%$ por peso). Igualmente se logró verificar que la relación $\mathrm{Ca} / \mathrm{P}$ aumentaba al adicionar carbonato a la estructura de la Hidroxiapatita hasta valores que pueden ser aptos para su aplicación como implante óseo.

\section{AGRADECIMIENTOS}

Al Grupo de Investigación en Materiales y Recubrimientos Cerámicos (GIMACYR) de la Universidad de Antioquia y al programa Jóvenes Investigadores UdeA por la pasantía en el proyecto Colciencias: Desarrollo de biomateriales compuestos de matriz cerámica para plataformas de crecimiento celular con liberación controlada de antibióticos, para aplicaciones como implantes óseos

\section{REFERENCIAS}

[1] Rodríguez Escobar, W., Bejarano Barrera, H., \& Villazón Amarís, H. (1999). Importancia estratégica de los nuevos materiales en el desarrollo sostenible y como alternativa de competitividad. Ciencia $\mathrm{E}$ Ingeniería Neogranadina, 8, 33-42. https://doi.org/10.18359/rcin.1408

[2] Wang, W., \& Yeung, K. W. K. (2017). Bone grafts and biomaterials substitutes for bone defect repair: A review. Bioactive Materials, 2(4), 224-247. 
https://doi.org/10.1016/j.bioactmat.2017.05. 007

[3] Roy, M., Bandyopadhyay, A., \& Bose, S. (2017). Ceramics in Bone Grafts and Coated Implants. In Materials and Devices for Bone Disorders. Elsevier Inc. https://doi.org/10.1016/B978-0-12-8027929.00006-9.

[4] Legeros, Racquel Zapanta, \& Legeros, J. P. (2008). Hydroxyapatite. Bioceramics and Their Clinical Applications, 367-394. https://doi.org/10.1533/9781845694227.2.36 7

[5] Botero, Y. (2016). Hidroxiapatita carbonatada, una opción como biomaterial para implantes: una revisión del estado del arte. Revista Colombiana de Materiales, No. 8, pp. 79-97. Revista Colombiana de Materiales, 8, 79-97.

[6] Londoño López, M., Echevarría, A., \& De La Calle, F. (2006). Características cristaloquímicas de la hidroxiapatita sintética tratada a diferentes temperaturas. Revista EIA, 5, 109-118.

[7] Landi, E., Celotti, G., Logroscino, G., \& Tampieri, A. (2003). Carbonated hydroxyapatite as bone substitute. Journal of the European Ceramic Society, 23(15), 2931-2937. https://doi.org/10.1016/S09552219(03)00304-2

[8] Lafon J-P (2004). Synthese, stabilite thermique et frittage d'hydroxyapatites carbonatees. Universite de Limoges.

[9] (Kamitakahara et al., 2015)Kamitakahara,
M., Nagamori, T., Yokoi, T., \& Ioku, K. (2015).

Carbonate-containing hydroxyapatite synthesized by the hydrothermal treatment of different calcium carbonates in a phosphate-containing solution. Journal of Asian Ceramic Societies, 3(3), 287-291. https://doi.org/10.1016/j.jascer.2015.05.002

[10] Vignoles M. Contribution à l'etude des apatites carbonatées de type $B$. Institut National Polytechnique de Toulouse, 1984.

[11] Legeros, R. Z., Legeros, J. P., \& Trautz, O. R. (n.d.). Effect of carbonate on the crystallinity. 57-66.

[12] Frank-Kamenetskaya, O. V. (2008). Structure, Chemistry and Synthesis of Carbonate Apatites - The Main Components of Dental and Bone Tissues. Minerals as Advanced Materials I, 241-252. https://doi.org/10.1007/978-3-540-771234_30

[13] Vignoles, M., Bonel, G., \& Bacquet, G. (1982). Etude physico-chimique des apatites carbonatees phospho-calciques semblables a la francolite. Bulletin de Mineralogie, 105(3), 307-311.

https://doi.org/10.3406/bulmi.1982.7621

[14] Safarzadeh, M., Ramesh, S., Tan, C. Y., Chandran, H., Ching, Y. C., Noor, A. F. M., Krishnasamy, S., \& Teng, W. D. (2020). Sintering behaviour of carbonated hydroxyapatite prepared at different carbonate and phosphate ratios. Boletin de La Sociedad Espanola de Ceramica y Vidrio, 59(2), 73-80. https://doi.org/10.1016/j.bsecv.2019.08.001 
[15] Kee, C. C., Ismail, H., \& Mohd Noor, A. F. (2013). Effect of synthesis technique and carbonate content on the crystallinity and morphology of carbonated hydroxyapatite. Journal of Materials Science and Technology, 29(8), 761-764. https://doi.org/10.1016/j.jmst.2013.05.016

[16] Ferguson J, Diefenbeck M, McNally M (2017). Ceramic Biocomposites as Biodegradable Antibiotic Carriers in the Treatment of Bone Infections. $J$ Bone $J t$ Infect. Available from: http://www.jbji.net/v02p0038.htm

[17] Fernández, M. M. (2010). Materiales compuestos nanoestructurados biocompatibles con matriz de hidroxiapatito. Psicothema, 11(003), 679-689.

[18] Venkateswarlu, K., Sandhyarani, M., Nellaippan, T. A., \& Rameshbabu, N. (2014).
Estimation of Crystallite Size, Lattice Strain and Dislocation Density of Nanocrystalline Carbonate Substituted Hydroxyapatite by $X$ ray Peak Variance Analysis. Procedia Materials Science, 5, 212-221. https://doi.org/10.1016/j.mspro.2014.07.260

[19] Xue, C., Chen, Y., Huang, Y., \& Zhu, P. (2015). Hydrothermal Synthesis and Biocompatibility Study of Highly Crystalline Carbonated Hydroxyapatite Nanorods. Nanoscale Research Letters, 10(1), 1-6. https://doi.org/10.1186/s11671-015-1018-9 $\mathrm{A}<\mathrm{a}<$ 